\title{
Electrically induced spin resonance fluorescence. II. Fluorescence spectra
}

\author{
A. Nogaret* and N. J. Lambert \\ Department of Physics, University of Bath, Claverton Down, Bath BA2 7AY, United Kingdom \\ F. M. Peeters \\ Department of Physics, University of Antwerp, Groenenborgerlaan 171, B-2020 Antwerpen, Belgium
}

(Received 21 February 2007; revised manuscript received 25 May 2007; published 8 August 2007)

\begin{abstract}
We model the fluorescence spectra of planar spin oscillators to find conditions that maximize spin resonance fluorescence. Spin oscillators perform Rabi oscillations under the effect of a periodic effective magnetic field caused by the winding motion of an electron in a gradient of magnetic field. We show that, despite the weak coupling of the spin magnetic dipole to the vacuum, spin oscillators excited by a direct current output a few nanowatts of microwave power, which is comparable to the best microwave sources. The large quantum efficiency relies on the combination of two effects. On the one hand, the spontaneous emission rate is enhanced by the synchronization of spin oscillators, which interact through the microwave field that they emit. On the other hand, the huge Rabi frequencies experienced by spin oscillators promote spins into upper levels of Zeeman transitions, from which a radiative cascade is triggered. We demonstrate different regimes of fluorescence which correspond to different values of the Rabi period relative to the spontaneous decay time and to the oscillator dwell time in the gradient of magnetic field. We investigate the device parameters which make these regimes experimentally accessible and find conditions that optimize microwave output. We find that microwave emission is centered around the cutoff frequency of spin oscillators. This has the advantage that the peak emission frequency may be tuned from zero continuously up to a few hundred gigahertz using an electrostatic gate. Quite remarkably for a spintronics effect, electrically induced spin resonance fluorescence does not require the injection of a spin polarized current. In fact, we show that microwave spectra are mostly independent of the incoming spin polarization except for magnetic waveguides which are shorter than a certain critical length, which we will specify.
\end{abstract}

DOI: $10.1103 /$ PhysRevB.76.075312

PACS number(s): 85.75. $-\mathrm{d}, 73.23 .-\mathrm{b}, 76.20 .+\mathrm{q}$

\section{INTRODUCTION}

Nanoscale microwave sources present great interest as a novel experimental test bench for magnetodipole interactions in quantum electrodynamics ${ }^{1}$ and for the integration of high speed cellular networks in microelectronics. ${ }^{2}$ One path toward microwave generation is to use current driven magnetization reversal ${ }^{3-5}$ to excite high frequency oscillations of the magnetization. Recent work by Kiselev et al. ${ }^{6}$ has shown that a spin polarized current traversing the free magnetic layer of a spin valve propels high frequency oscillations of the magnetization through the transfer of a magnetic torque. Individual nanopillars have demonstrated a few nanowatts of microwave output power. They have also shown that the resonant frequency depends on the applied current, which provides a useful means of controlling the oscillator frequency. Synchronization between spin torque oscillators has been investigated with the aim of enhancing the output power. ${ }^{7-10}$ A tenfold increase in microwave intensity and coherence has been obtained by coupling two oscillators and driving them in the phase locked mode. Theoretical investigations of magnetic nanocontacts interacting via spin waves ${ }^{11}$ or stimulated microwave currents ${ }^{12}$ have shown that self-phase locking occurs because magneto-dipole interactions are strongly nonlinear. It is also believed that the magnetization of the free layer follows complex trajectories which are sensitive to the magnetic field and explain the observation of distinct dynamic regimes in a narrow current range. ${ }^{13-15}$ The generation of stable oscillations, therefore, requires precise current conditions, which may complicate the implementation of spin valves as broadband microwave emitters.

Microwave emission has also been detected in molecular magnets of manganese acetate. ${ }^{16,17}$ This system is well known for its magnetization reversal dominated by quantum tunneling of the magnetization. If, however, the magnetic field is swept rapidly, the Zeeman energy levels of the molecule cross without coupling, leading to population inversion in the spin ladder. ${ }^{18}$ The system then returns to equilibrium by emitting a single burst of photons at a wavelength corresponding to the Zeeman energy gap. ${ }^{19}$ Although the magnetodipole emission rate of a single spin is quite small, the coherent spontaneous emission of a large number of spins by a crystal of magnetic molecules ${ }^{20-22}$ has produced microwave bursts peaking at a few tens of femtowatts. ${ }^{23,24}$

Other magnetodipole microwave sources include notably magnetic dust in interstellar clouds, which is responsible for the so-called cosmic microwave foreground. ${ }^{25,26}$

In the present paper, we model the microwave fluorescence emanating from electrons confined both by the electrostatic potential of a quantum well and a gradient of magnetic field pointing out of the plane. The magnetic field gradient channels electrons in open orbits, ${ }^{19,27-36}$ which subject the electron spin to a periodic effective magnetic field. The frequency of this magnetic field is given by the electron oscillations about the contour of zero magnetic field. It increases with the angle at which the electron orbit cuts this contour. Small angles, $\theta \approx 0$, correspond to fast, small amplitude oscillations, whereas large angles, $\theta \approx 180^{\circ}$, correspond to slow, large amplitude, anharmonic oscillations. A homo- 
geneous magnetic field applied in the plane of the quantum well-i.e., perpendicular to the ac effective magnetic fieldinduces spin resonance when its Larmor frequency $\omega_{0}$ equals the oscillator frequency $\omega$. Under the drive of a current bias, the spin flips back and forth between the ground spin state and the excited state while drifting in the magnetic field gradient. These Rabi oscillations are responsible for the fluorescence of the spin resonance because they periodically promote the spin into the excited spin state, from which radiative decay occurs. Here, spin oscillators are excited by the direct longitudinal current carried by open orbits. The preceding paper ${ }^{37}$ has described in detail how photons are sequentially emitted at time intervals correlated with the period of Rabi oscillations, and how this triggers a radiative cascade, enabling one spin oscillator to output a large number of photons. Here, we report numerical calculations of spin resonance fluorescence that predict the microwave power generated by real devices. We show that, among all oscillators trapped in the gradient of magnetic field, some will fulfill conditions for emitting in the exponential regime, whereas others will fall in the Rabi regime and radiate efficiently. Among the latter, those with the smallest amplitude are prevented from radiating efficiently by the finite length of the waveguide. This occurs when the Rabi period is larger than the time span between the moment the spin enters the waveguide and the time it exits. By contrast, orbits with large oscillation amplitude will experience a large periodic magnetic field and complete hundreds of Rabi cycles before exiting the waveguide. This third category of orbits will radiate in the regime of radiative cascade, which is accompanied by bright emission. We compute microwave emission spectra for different initial conditions and device parameters. We find that these parameters produce subtle changes in the delicate structure of microwave output spectra, which results from the competition and interplay between the three radiative modes.

Microwave sources based on planar spin oscillators are useful for generating continuous wave microwave power. We find that the power is in the range of a few nanowatts, which is comparable to the output of spin torque oscillators. ${ }^{6}$ Based on our modeling, planar spin oscillators, however, require hundred times less input current to generate the same amount of output power. From a fundamental point of view, spin oscillators are the first physical system for which a steady state of super-radiant emission is stabilized by the constant regeneration of top spin state. This is in contrast to optically pumped masers ${ }^{20}$ and Rydberg atoms ${ }^{21}$ that radiate by short bursts. Our results show that the output power can be adjusted with a great amount of flexibility through the choice of material and structural parameters. The calculated spectra show a single well defined emission line, which dominates the microwave background and behaves as a monochromatic source of linewidth $\omega_{c} / 10$ or $\approx 30 \mathrm{GHz}$. The peak frequency is continuously tunable in the $0-500 \mathrm{GHz}$ frequency range by means of an electrostatic gate.

The paper is organized as follows. Section II describes the radiative cascade at resonance. We discuss the asymptotic behavior of the fluorescence when the electron dwell time in the waveguide is much longer or much shorter than the radiative lifetime. We discuss the saturation of the fluorescence when the Rabi frequency is either much larger or much smaller than the rate of spontaneous decay. We then detune the photon frequency with respect to the Zeeman energy gap to obtain the emission lines in the Rabi and exponential regimes. Section III reports on the microwave spectra and the microwave power emanating from open orbits trapped by one line of zero magnetic field. We also describe their dependence on the length of the magnetic waveguide, the magnetic gradient, and the spin polarization of the incoming current. Section IV discusses the influence of temperature and material parameters on real devices. Section V summarizes our findings and presents our conclusion.

\section{RADIATIVE CASCADE}

The intensity of the spin resonance fluorescence depends on the frequency at which the spin occupies the high energy level, since this is the one from which radiative decay occurs. For Rabi oscillations to occur, two conditions must be realized simultaneously. Firstly, the oscillator frequency must be resonant with the Larmor frequency, which is the condition for spin resonance. Secondly, the Rabi frequency $\Omega_{1}$ has to be higher than the rate of spontaneous decay, $\Gamma_{s r} / 2$, otherwise, the excited state would only decay by the spontaneous emission of a photon. When both conditions are satisfied, the probability of photon emission oscillates at the Rabi frequency with some exponential damping due to the coupling to the field. After a photon has been emitted, the phase memory of previous Rabi oscillations is lost and a new series of damped oscillations restart from the ground state. From the time an electron enters the waveguide to the time it leaves, its spin may have gone through this process many times, each time emitting one photon. We have calculated the probabilities for the sequential emission of one, two, up to an infinite number of photons in Eqs. (45)-(50) of the preceding paper. ${ }^{37}$ The power generated by this cascade emission [Eq. (60)] grows rapidly because the probability of many photon processes increases with the length of the waveguide, and these add to the more likely processes involving fewer photons. An increase in Rabi frequency will amplify cascade emission as a higher spin transfer rate into the excited state makes radiative decay more likely. The efficiency of cascade emission increases as the average time interval between radiative events is shortened.

By contrast, if radiative emission is faster than the Rabi frequency, $\Gamma_{s r} / 2 \gg \Omega_{1}$, spins prepared in the upper energy level will decay exponentially to the ground state soon after they have entered the magnetic waveguide. This decay takes place on a time scale of $2 / \Gamma_{s}$, during which spin flip oscillations are strongly damped. This exponential radiative regime is transient and non-self-regenerating, which generates microwaves in a localized area at the entrance of the waveguide.

Figure 1 shows the fluorescence power generated by a spin oscillator as a function of the dwell time in the magnetic waveguide, $\tau_{d w e l l}$. The power is normalized so as to give a direct reading of the number of photons emitted per spin oscillator. We introduce the dimensionless parameter $r$ $=\Omega_{1} /\left(\Gamma_{s r} / 2\right)$ to study the transition from the exponential re- 


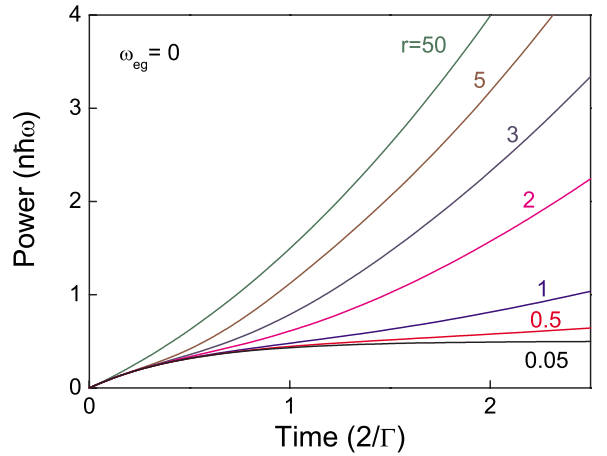

FIG. 1. (Color online) Electromagnetic power radiated at resonance as a function of the electron dwell time in the gradient of magnetic field for different values of $r=\Omega_{1} /\left(\Gamma_{s r} / 2\right)$, i.e., the Rabi frequency to the spontaneous decay rate. The power is expressed in units of the photon energy, $\hbar \omega$, times the rate $n$ at which electrons are injected in the device. The plot is for resonant conditions when the detuning of the photon frequency to the Larmor frequency is $\omega_{e g}=\omega-\omega_{0}=0$ ( $e$, "excited state;" $g$, "ground state"). The direct current passing through the waveguide has no initial spin polarization.

gime $(r<1)$ to the Rabi regime $(r>1)$. When $r \ll 1$, the fluorescence power increases from zero at small dwell times and then saturates at a power level of $n \hbar \omega / 2$ when $\tau_{d w e l l}$ $\gg 2 / \Gamma_{s r}$. Beyond $2 / \Gamma_{s r}$, the output power does not increase further, demonstrating that microwave emission, in the exponential regime, mostly takes place at the entrance of the waveguide. Spins initially prepared in the excited state decay radiatively, whereas spins prepared in the ground state do not couple to the field and give no contribution to the fluorescence. To calculate the fluorescence power plotted in Fig. 1, we have assumed that the current is injected into the waveguide with no spin polarization. This explains why the power saturates at an average of $1 / 2$ photon emitted per spin as only one spin out of two is prepared in the excited state. Our model, therefore, predicts that fluorescence will be localized within a length scale of $\approx 2 \pi v_{F} / \Gamma_{s r}$ at the entrance of the magnetic waveguide.

When the Rabi frequency and the spontaneous rate of decay become comparable, $r=1$, spins are more likely to flip into the excited state within the radiative lifetime. This initiates the radiative cascade. Figure 1 shows that the fluorescence power increases rapidly with $r$ and eventually reaches a saturation value when $r \gg 1$. This saturation corresponds to the $r=50$ curve when the radiative cascade is fully developed and multiple photons are emitted per spin oscillator. For example, at $\tau_{d w e l l}=2\left(2 / \Gamma_{s r}\right)$, the average spin oscillator emits four photons. Unlike in the exponential regime, here both spin up and spin down electrons participate in the radiative cascade so that initial spin polarization is irrelevant. In addition, the number of photons emitted per spin oscillator increases with increasing dwell time. This makes clear that radiative emission delocalizes along the waveguide. Interestingly, the superlinear increase in the $r=50$ curve suggests that the photon emission rate per unit length of the waveguide actually increases. Fluorescence near the exit of the waveguide will be brighter than near its entrance. This effect is explained by the rising probability of multiple photon

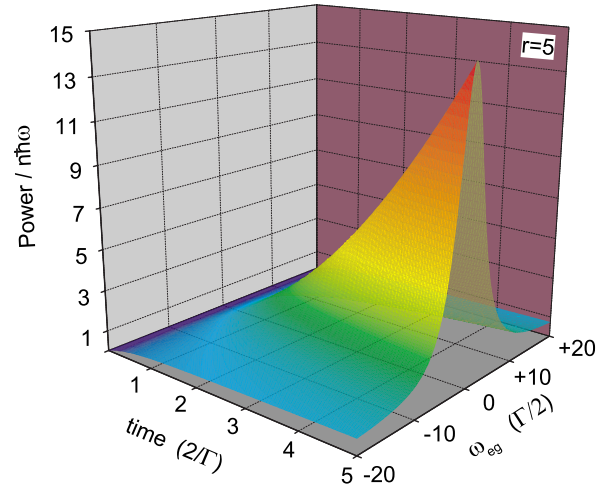

FIG. 2. (Color online) Fluorescence power plotted as a function of the oscillator dwell time in the magnetic field gradient and the detuning $\omega_{e g}$ of the photon frequency relative to the resonant frequency. The calculation is done in the Rabi regime: $r=5$.

emission processes when spin oscillators dwell longer in the magnetic field gradient. Hence longer waveguides are desirable to maximize the output power from the radiative cascade. The waveguide length $L$ is related to the oscillator dwell time through $\tau_{d w e l l} \approx L /\left(\pi v_{F}\right)$.

The fluorescence plot shown in Fig. 1 is a universal result, where the only arbitrary input is the spin polarization of the incoming current. In a gradient of magnetic field, oscillators swing with different amplitudes depending on the angle $\theta$ at which the open orbit cuts the contour of zero magnetic field. Their spins experience different magnetic field amplitudes, hence different strengths of the Rabi coupling. The fastest oscillators, for which $\theta \approx 180^{\circ}$, sense a small effective magnetic field and radiate in the exponential regime. By contrast, slower oscillators, with wider swing, sense a larger ac magnetic field, and hence, radiate in the Rabi regime. The boundary between the exponential regime and the Rabi regime $(r$ $=1$ ) gives the following criterion on the amplitude of oscillations:

$$
Z_{1}=\frac{\Gamma_{s r}}{2 \gamma b l_{b}} .
$$

Here, $\gamma$ is the gyromagnetic ratio of the electron spin, $l_{b}$ is the maximum amplitude of oscillations, and $Z_{1}$ is the maximum swing of exponentially decaying oscillators expressed in units of $l_{b}$. At $500 \mathrm{GHz}$ (least favorable conditions for Rabi oscillations), $\Gamma_{s r}=3.93 \times 10^{6} \mathrm{~s}^{-1}$, and $l_{b}=323 \mathrm{~nm}$, we find $Z_{1}=1.7 \times 10^{-5}$. Using $Z=\cos (\theta / 2),{ }^{27}$ we find $\theta_{1}$ $=179.998^{\circ}$ to be the angle separating Rabi oscillators at small values of $\theta$ from exponential oscillators at $\theta>\theta_{1}$. From this result, we conclude that the majority of spin oscillators radiate in the Rabi regime.

Figures 2 and 3 map the spin resonance fluorescence in the Rabi and exponential regimes, respectively, when the photon frequency is detuned from resonance. The fluorescence peaks in these plots have different linewidths and amplitudes, which we now discuss. In Fig. 2, the resonance is Lorentzian, with a linewidth given by $\sqrt{\Gamma^{2} / 4+\Omega_{1}^{2}}$. For $r \gg 1$, this approximates to the Rabi frequency $\Omega_{1}$. At resonance, $\omega_{e g}=0$, the fluorescence power shows a superlinear increase, 


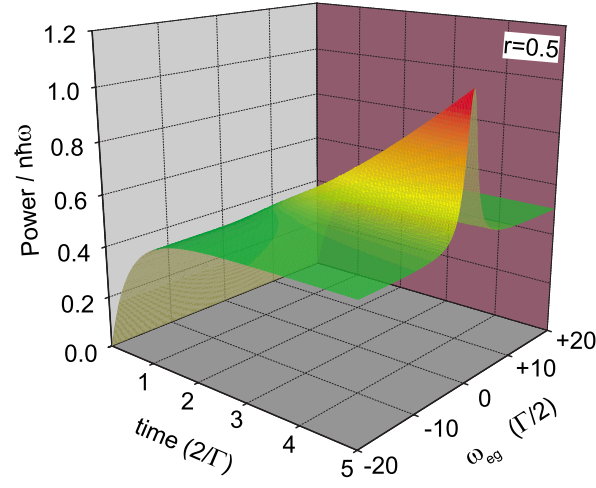

FIG. 3. (Color online) Fluorescence power plotted as a function of the oscillator dwell time in the magnetic field gradient and the detuning $\omega_{e g}$ of the photon frequency relative to the resonant frequency. The calculation is done at the threshold of the exponential regime: $r=0.5$.

which is the signature of the radiative cascade, and at $\tau_{d w e l l}$ $=5\left(2 / \Gamma_{s r}\right)$, each oscillator emits an average of 14 photons. If we turn to the off-resonance fluorescence power and follow its variation along the edge of the plot at $\omega_{e g}=-20$, we observe an initial increase in fluorescence power up to $2 / \Gamma_{s}$. For longer dwell times, the output power increases very little and saturates at a value of 0.8 photons per oscillator. This can be explained in the following way. At off-resonance, the occupancy of the excited state decreases despite spin flip attempts being made at a faster rate. As a result, the frequency of spontaneous decay decreases, which has the effect of switching off the radiative cascade. The fluorescence power emitted off-resonance in the Rabi regime, therefore, appears similar to the power emitted in the exponential regime even though the underpinning physical mechanisms are different. Namely, the off-resonant decay of Rabi oscillations will occur whether radiative coupling is present or not, whereas exponential decay requires radiative coupling. Figure 3 shows the fluorescence in the exponential regime. The linewidth now approximates to the rate of radiative decay, $\Gamma_{s r} / 2$, while the off-resonance power shows a saturation plateau at $1 / 2$. The resonant power at $\omega_{e g}=0$ is 12 times weaker than the resonant power in the Rabi regime of Fig. 2. If one calculates the fluorescence power for $r \ll 1$, we find the resonant peak to be absorbed by the nonresonant background. The comparison of Figs. 2 and 3 hence demonstrates the power amplification and bandwidth of the radiative cascade.

\section{MICROWAVE EMISSION SPECTRA}

The fluorescence spectrum of a magnetic waveguide is a superposition of the electron spin resonance lines calculated in the previous section. For an individual spin oscillator, these lines are centered on the fundamental frequency and each of its harmonic frequencies. A spin oscillator is part of a bundle of orbits which are parametrized by angle $\theta$. The bundle cover a continuous range of fundamental frequencies, $0-\omega_{c}$, where $\omega_{c}$ is the frequency of the fastest orbits. ${ }^{27}$ Therefore the microwave emission spectra of real waveguides will appear as the frequency dispersion curve of

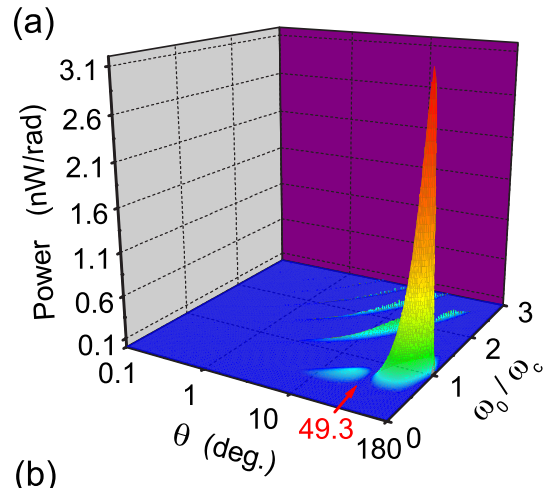

(b)

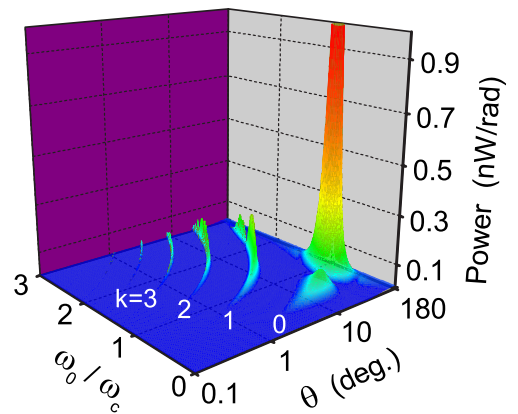

FIG. 4. (Color online) Microwave power emitted by individual open orbits $(\theta)$ as a function of the Larmor frequency $\left(\omega_{0}\right)$. The Larmor frequency is expressed in units of the cutoff frequency of spin oscillators, which is $\omega_{c}=2.5 \times 10^{12} \mathrm{~s}^{-1}$ or $400 \mathrm{GHz}$. (a) and (b) show the same plot with different vertical scales and under different angles. The former gives the peak power and linewidth, while the latter shows the finer structure, which is discussed in the text. Parameters: current $I=10 \mu \mathrm{A}$, electron effective mass $m^{*}=0.023 m_{0}$ (InAs), Landé factor $g=-15$ (InAs), electron density $n_{s}=1$ $\times 10^{11} \mathrm{~cm}^{-2}$, magnetic gradient $b=2 \times 10^{6} \mathrm{~T} / \mathrm{m}$, length of magnetic waveguide $L=1000 \mu \mathrm{m}$, no incoming spin polarization $n_{g}(t$ $=0)=n_{e}(t=0)=0.5$, and number of spins oscillating in phase $N$ $=10^{10}$.

the bundle, with satellite curves at odd multiples of the fundamental frequency. The spectra in Fig. 4 display the fluorescence power output by individual orbits as a function of the Larmor frequency. The numerical model assumes an InAs quantum well, with the parameters given in the caption of Fig. 4. Based on these values, we calculate $\Gamma_{s r}, \Omega_{1}$, and $\tau_{d w e l l}$ to relate the various modes of emission to actual physical quantities.

At frequencies between 100 and $500 \mathrm{GHz}$, the wavelength is in the millimeter range. This defines an area of the two-dimensional electron system containing $N \approx 10^{10}$ electrons, where their magnetic dipoles radiate simultaneously through coherent spontaneous emission or super-radiance. Coherent emission enhances the bare radiation rate, $\Gamma$ $=3.93 \times 10^{-4} \mathrm{~s}^{-1}(500 \mathrm{GHz})$, by a factor equal to the number of coupled oscillators ${ }^{38}$ to $\Gamma_{s r}=N \Gamma=3.93 \times 10^{6} \mathrm{~s}^{-1}$. The $N$ oscillators have the same frequency and move at the same velocity, which maintain their coherence. ${ }^{20}$ It follows that the time scale of exponential decay is $2 / \Gamma_{s r}=250 \mathrm{~ns}$, which corresponds to a decay length in the waveguide of approximately $35 \mathrm{~mm}$. Spin oscillators experience magnetic fields ranging between 0 and $0.65 \mathrm{~T}$, hence have Rabi frequencies between 0 and $110 \mathrm{GHz}$. We already know from Eq. (1) that 
most oscillators within this range belong to the Rabi regime, $r>1$. One must also keep in mind that the waveguide has finite length, which limits the number of cycles of Rabi oscillations effected within the region where the magnetic field gradient is applied. Because the radiative decay length of $35 \mathrm{~mm}$ exceeds the device dimensions, a fraction of the orbits in the Rabi regime are unable to complete one full Rabi cycle before exiting the waveguide. This can be seen in the following example. In the $1000 \mu \mathrm{m}$ long waveguide, the average dwell time is $\tau_{d w e l l} \approx 8 \mathrm{~ns}$. Within this time, oscillators with maximum amplitude, $l_{b}$, flip $\Omega_{1} \tau_{\text {dwell }}=880$ times. At the other end of the spectrum, an oscillator will complete at least one Rabi cycle if its amplitude is larger than

$$
Z_{2}=\frac{1}{\gamma b l_{b} \tau_{d w e l l}} .
$$

This corresponds to $\theta_{2}=2 \arccos \left(Z_{2}\right)=179.87^{\circ}$. Hence, still a majority of spin oscillators- $0<\theta_{2}<179.87^{\circ}$ - have enough time to complete more than one spin flip. The above estimates have given us an order of magnitude of the length scales of the problem and will enable us to interpret the microwave emission spectra, which we are now going to present.

\section{A. Fan structure}

Figure 4(a) shows the spectrum emitted by a $1 \mathrm{~mm}$ long waveguide. This depicts a fan structure for which each branch corresponds to the excitation of a mode of the spin oscillator by the Larmor frequency. Each mode is labeled by an index $k$ in Fig. 4(b). The $k=0$ branch corresponds to the resonance with the fundamental mode of the individual oscillators: $\omega_{0}=\omega(\theta)$. The $k=1,2,3, \ldots$, branches show successive resonances with odd oscillator harmonics as the Larmor frequency increases. The resonant condition here is $\omega_{0}=(2 k$ $+1) \omega(\theta)$, each branch de facto maps the $\omega(\theta)$ dispersion curve of the spin oscillators. ${ }^{27}$ The $k=0$ branch terminates at $\omega_{0}=\omega_{c}$ at the frequency of the fastest oscillators. Similarly, the $k=1$ branch terminates at $3 \omega_{c}$, etc.

The main feature of interest in the spectrum is the large peak centered at coordinates $\left(150^{\circ}, \omega_{c}\right)$ near the top end of the $k=0$ branch. At this point, the power peaks at $3.1 \mathrm{nW} / \mathrm{rad}$ and the half width at half maximum is $\approx 30 \mathrm{GHz}$. The bandwidth is sufficiently narrow to be considered as quasimonochromatic. Emission localizes at the top end of the $k=0$ branch, because oscillators near $\omega_{c}$ are supported by fast drifting open orbits, which carry a large fraction of the excitation current. The second reason is the magnetodipole radiative rate growing as the cube of the photon frequency, enhancing the fluorescence in each branch as $\theta$ increases. The drop in power from the peak at $\theta \approx 150^{\circ}$ to the edge of the map at $180^{\circ}$ is the radiative cascade switching off, following the argument that we have developed above. Namely, we know that orbits above $\theta>179.87^{\circ}$ do not radiate efficiently either due to evanescent decay in the exponential regime [Eq. (1)] or due to the finite length of the waveguide [Eq. (2)].

The fan structure in Fig. 4(b) vanishes at $\theta=49.3^{\circ}$, because this angle corresponds to the formation of " 8 " shaped

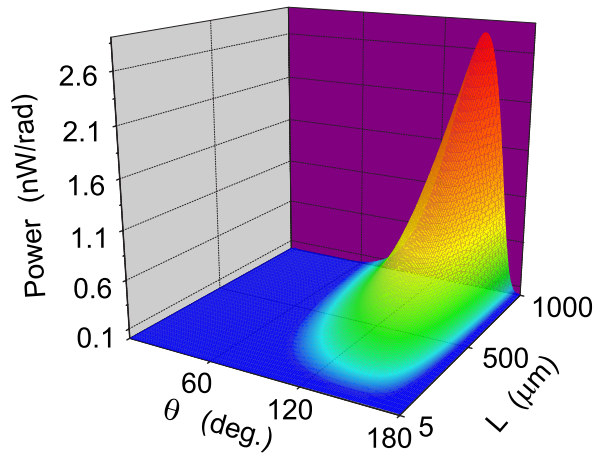

FIG. 5. (Color online) Microwave emission spectrum calculated as a function of the length of the magnetic waveguide. We have set $\omega_{0}=\omega_{c}$ to show the dependence of the peak power. Other parameters are the same as those in Fig. 4.

orbits, which are stationary. For $\theta>49.3^{\circ}$, microwave emission is excited by a positive current, whereas for $\theta<49.3^{\circ}$, it is excited by a negative current.

\section{B. Variation of the waveguide length}

The effect of increasing the length of the magnetic waveguide is shown in Fig. 5. The plot demonstrates the evolution of the system from a regime of weak fluorescence at short dwell times to a regime of intense fluorescence supported by the onset of the radiative cascade. With a length $L$ $=1000 \mu \mathrm{m}$, the waveguide is sufficiently long for the spin to flip hundreds of times before exiting. By contrast, the output power of the $5 \mu \mathrm{m}$ long waveguide is negligible despite the system being tuned at resonance. This dependence emphasizes the importance of the electron dwell time in the waveguide for generating large fluorescence power.

\section{Dependence on the magnetic gradient}

We next turn to the dependence of the output power on the gradient of the magnetic field, $b$, which is shown in Fig. 6 . The effect of increasing the gradient of magnetic field is to reduce the amplitude of electron oscillations as $b^{-1 / 2}$ and to increase the amplitude of the effective periodic magnetic field ${ }^{27}$ as $b^{1 / 2}$. The range of curvature radii at different points of an electron orbit widens. As a result, the anharmonicity of oscillations increases, which enhances the intensity of the harmonic peaks relative to the main peak. The $k=1,2,3, \ldots$, branches, which are very faint in Fig. 6(a), become comparable to the $k=0$ resonant peak in Fig. 6(b) after a fourfold increase in the magnetic field gradient.

The magnetic field gradient also modifies the cutoff frequency $\omega_{c}$, which varies as $\propto b^{1 / 2}$. This explains why the peak frequency increases from $275 \mathrm{GHz}$ in Fig. 6(a) to $550 \mathrm{GHz}$ in Fig. 6(b). Another notable feature is the shift in fluorescent emission toward the top end of the $k=0$ branch as the magnetic field gradient becomes steeper. The peak is displaced from $\theta=153^{\circ}$ to $\theta=160^{\circ}$, while its amplitude increases fivefold from 0.47 to $2.89 \mathrm{nW} \mathrm{rad}{ }^{-1}$. These changes are caused by the increase in Rabi frequency. On the one hand, an increase in $b$ increases $r$, with the effect of increas- 
(a)

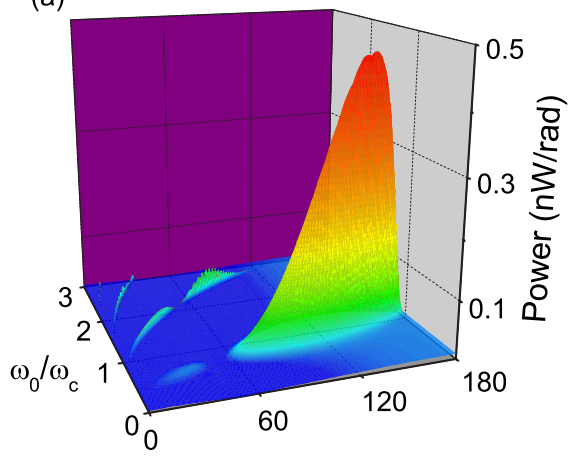

(b)

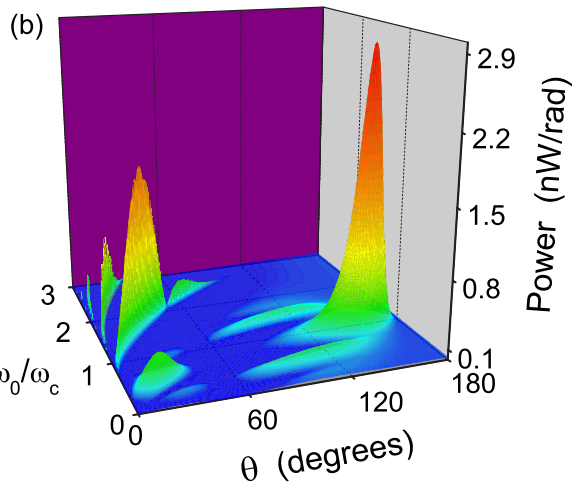

FIG. 6. (Color online) Dependence of fluorescence on the gradient of magnetic field. The magnetic field gradient is $b=1$ $\times 10^{6} \mathrm{~T} \mathrm{~m}^{-1}$ in (a) and $b=4 \times 10^{6} \mathrm{~T} \mathrm{~m}^{-1}$ in (b).

ing the power emitted at resonance as shown in Fig. 1. On the other hand, the conditions stipulated by Eqs. (1) and (2) are relaxed when the product $b l_{b} \propto b^{1 / 2}$ increases. Consequently, oscillators with $\theta$ closer to $180^{\circ}$ move into the Rabi regime with the effect of shifting the peak to higher $\theta$.

In Fig. 4(b), we see up to two additional sidebands above and below the $k=0$ branch. These are fingerprints of damped Rabi oscillations performed by the spins injected from the top energy level. For these spins, interactions with the electromagnetic field confer a higher amplitude to off-resonant Rabi oscillations than to resonant ones. This effect is shown clearly in Fig. 7 of the preceding paper. ${ }^{37}$

In summary, increasing the magnetic field gradient changes the emission spectra in subtle ways. Its main effects are to shift the peak emission to higher frequency and to increase the peak amplitude.

\section{Effects of spin polarization}

The fluorescence spectra that we have calculated so far have assumed a driving current without spin polarization. Here, we investigate spin polarized currents and show that microwave emission rapidly becomes independent of the initial spin polarization on distance shorter than the exponential decay, $2 \pi v_{f} / \Gamma_{s r}$.

Figure 7 shows the dependence of the microwave spectrum of the injected spin polarization for two lengths of waveguide, respectively, 1000 and $10 \mu \mathrm{m}$. In Fig. 7(a), the emission peak at $\theta \approx 150^{\circ}$ drops from $2.97 \mathrm{nW} / \mathrm{rad}$ at $n_{e}$ $=1$ to $2.74 \mathrm{nW} / \mathrm{rad}$ at $n_{e}=0$, and is quasi-independent of the
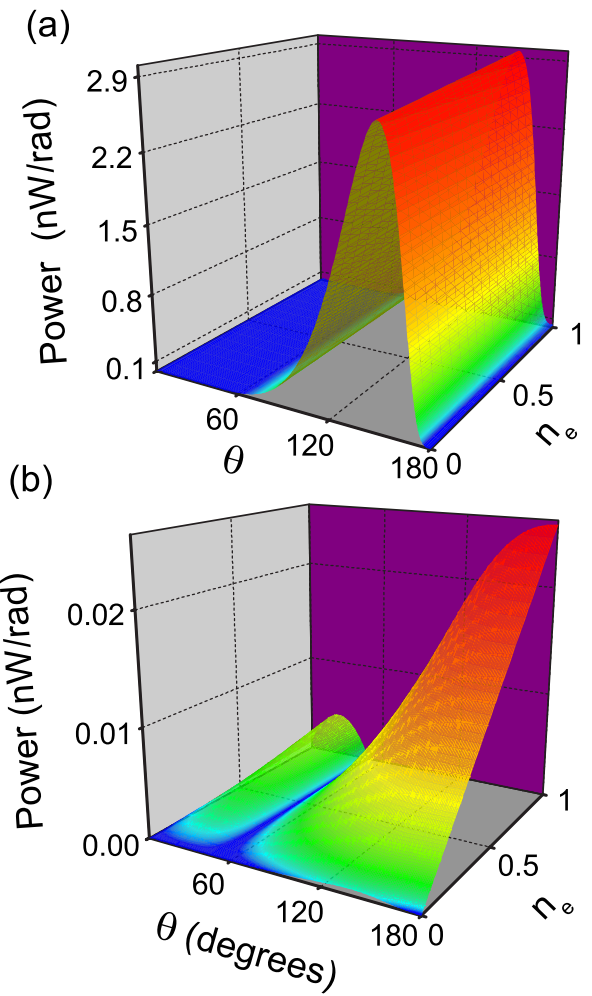

FIG. 7. (Color online) Dependence of the fluorescence spectrum on the spin polarization of the incoming current. $n_{e}$ represents the fraction of spins prepared in the high energy level. (a) is calculated for a $1000 \mu \mathrm{m}$ waveguide and (b) for a $10 \mu \mathrm{m}$ waveguide.

initial spin polarization. This indicates that almost all spin oscillators have decayed radiatively at least once and, by the time electrons exit the waveguide, the memory of the initial spin polarization is almost completely lost. After the first radiative event, spins injected from either state of the electronic transition restart their oscillations at random times and from the same low energy state; consequently, they contribute equally to the fluorescence signal.

In contrast, Fig. 7(b) shows the power output by the short waveguide to be proportional to the spin polarization. The output power is zero at $n_{e}=0$, and this increases to $0.026 \mathrm{~mW} / \mathrm{rad}$ at $n_{e}=1$ and $\theta=180^{\circ}$. The explanation is simple. Spins prepared in the top spin state before entering the waveguide are the only ones decaying radiatively. Spin prepared in bottom energy state cannot radiate nor have enough time to flip into the top energy state. We, therefore, estimate that the length scale over which spin memory is lost in the radiative cascade is intermediate between the two plots, that is, of the order of $100 \mu \mathrm{m}$. This is much less than the length of radiative decay, $35 \mathrm{~mm}$. It is, therefore, an attractive aspect of this spintronics effect that no initial spin polarization is required, nor is it desirable for maximizing the output power.

\section{E. Power output}

Figure 8 shows the power output, integrating the contribution of all oscillators as a function of the Larmor frequency. For positive currents, the power peaks at $1.2 \mathrm{nW}$. 


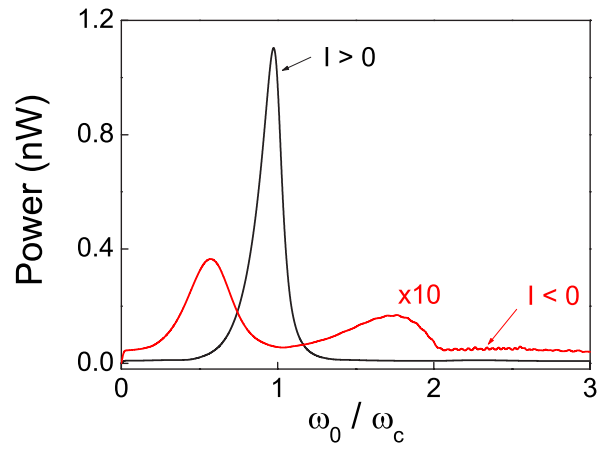

FIG. 8. (Color online) Microwave power emitted by the whole distribution of open orbits as a function of the Larmor frequency. Positive currents flow with open orbits for which $49.3^{\circ}<\theta$ $<180^{\circ}$, whereas negative currents correspond to $0<\theta<49.3^{\circ}$. Device parameters are the same as in Fig. 4.

The peak position is centered on the cutoff frequency of oscillators and has half width at half maximum of $\Delta \omega$ $\approx 0.12 \omega_{c}$. For negative current, the power peaks at a lower frequency, $\omega_{0} \approx 0.5 \omega_{c}$, because spin oscillators are slower for smaller values of $\theta$. For this reason also, magnetodipole coupling is weaker, which explains why the peak power output is more than a magnitude smaller than for positive current. Higher harmonics contribute to the satellite peak seen at $\omega_{0} \approx 1.8 \omega_{c}$.

We have, therefore, shown that peak output power of the order of nanowatts is theoretically possible. The emission line is well resolved and the peak frequency is centered on $\omega_{c}$. Since the peak frequency is $\propto n_{s}^{1 / 4}$, magnetic waveguides present the advantage of generating frequencies in the range of $0-500 \mathrm{GHz}$, which one controls with an electrostatic gate.

\section{DISCUSSION}

Electrically induced spin resonance fluorescence is observable in any material system provided it hosts a twodimensional electron system immersed in the appropriate magnetic field profiles. Maximizing the power of spin resonance fluorescence requires materials with high Landé $g$ factor. This is, firstly, because the rate of spontaneous decay depends on the square of the magnetic dipole moment. Secondly, large Landé $g$ factors allow spin resonance to be observed at moderately small magnetic fields. This is the reason why we have modeled magnetic waveguides based on InAs quantum wells.

The power output may be enhanced by embedding the magnetic waveguide in an electromagnetic cavity. ${ }^{24,39}$ On the one hand, a discrete photon density of states would enhance magnetodipole coupling for specific frequencies. On the other hand, photons present in the cavity would augment the ac effective magnetic field and enhance the efficiency of the radiative cascade.

Our calculation was done at zero temperature and assumed the system of spins to be in thermal equilibrium when they are all condensed in the ground energy level. At finite temperature, the smaller difference in occupancy of the ground and excited levels will attenuate radiative relaxation in the exponential regime. In contrast, the radiative cascade will be unaffected as it is insensitive to initial spin conditions. One expects the nonradiative relaxation rate to broaden the main emission line if it becomes larger than the Rabi frequency. Room temperature spin relaxation times for the following quantum wells: InAs (5.2 ps), ${ }^{40} \mathrm{GaAs}$ (32 ps), ${ }^{41}$ $\mathrm{ZnCdSe}$ (300 ps) (Ref. 42) are generally larger than the smallest Rabi period $\sim 2.5$ ps. Spin relaxation is, therefore, likely to broaden the fluorescence peak of InAs quantum wells at $300 \mathrm{~K}$, but its effect will be less important in GaAs and $\mathrm{ZnCdSe}$. Hence, our model should make reasonably accurate predictions of the peak power and the emission linewidth.

Planar spin oscillators have reminded several people of undulators which were proposed as x-ray sources in the $1980 \mathrm{~s} .{ }^{43}$ For the sake of putting spin oscillators in context, we now compare and contrast the properties of both systems. Undulators are multipole magnetic devices inserted in synchrotron rings that bend the electron beam in sinusoidal trajectories. The resulting bremsstralung radiation produces a bright coherent emission cone in the forward direction along the beam. ${ }^{44}$ Common to both undulators and spin oscillators is that both use inhomogeneous magnetic fields to produce oscillatory trajectories. For spin oscillators, a magnetic potential alone is not sufficient. A two-dimensional electrostatic confinement is essential to prevent the deflection of electrons by the Larmor field. In undulators, the Larmor magnetic field is zero (by symmetry), hence spin resonance does not occur. If one such field were applied, its primary effect would be to deflect the electron beam off its axis. A three-dimensional system such as an undulator cannot support the formation of spin oscillators. We also believe that the fluorescence of spin oscillators is unlikely to lead to lasing according to the model of the free electron laser (implemented by undulators). ${ }^{44,45}$ Lasing would require commensurability of the orbit wavelength to the wavelength of the electromagnetic radiation to sustain constructive interferences in the manner of a periodic diffraction grating. ${ }^{45}$ Instead, we have shown that bursts of spontaneous emission are correlated with the period of Rabi oscillations rather than the period of spin oscillators. Unlike undulators which require beams of relativistic electrons, spin oscillators function well at the more moderate Fermi velocities of semiconductors and metals. The flexibility in tuning electronic and photon cavities in semiconductor heterostructures holds an infinite potential for probing magnetodipole emission processes and for making nanoscale microwave sources.

\section{CONCLUSION}

We have numerically calculated the fluorescence of spin oscillators excited by a direct current flowing through a gradient of magnetic field. The efficiency of the microwave power depends on a subtle interplay between the Rabi frequency, the magnetodipole radiative rate, and the electron dwell time in the magnetic waveguide. We have found spin resonance fluorescence to be considerably enhanced by super-radiant coupling and by radiative cascade. The later effect requires strong Rabi coupling, which is easily obtained 
in real devices, and sufficiently long waveguides to let Rabi oscillations develop. Our results predict power output of $-60 \mathrm{~dB} \mathrm{~m}$, which is superior to the Johnson noise $(-174 \mathrm{~dB} \mathrm{~m})$ and makes this microwave source an attractive proposition for broadcasting.

\section{ACKNOWLEDGMENTS}

This work is supported by EPSRC (UK) and the Flemish Science Foundation (FWO-VI). *a.r.nogaret@bath.ac.uk

${ }^{1}$ B. Odom, D. Hanneke, B. D’Urso, and G. Gabrielse, Phys. Rev. Lett. 97, 030801 (2006).

${ }^{2}$ M.-C. Chang, V. Roychowdhury, L. Zhang, H. Shin, and Y. Qian, Proc. IEEE 89, 456 (2001).

${ }^{3}$ J. A. Katine, F. J. Albert, R. A. Buhrman, E. B. Myers, and D. C. Ralph, Phys. Rev. Lett. 84, 3149 (2000).

${ }^{4}$ W. H. Rippard, M. R. Pufall, S. Kaka, T. J. Silva, S. E. Russek, and J. A. Katine, Phys. Rev. Lett. 95, 067203 (2005).

${ }^{5}$ W. H. Rippard, M. R. Pufall, S. Kaka, S. E. Russek, and T. J. Silva, Phys. Rev. Lett. 92, 027201 (2004).

${ }^{6}$ S. Kiselev, J. Sankey, L. Krivorotov, N. Emley, R. Schoelkopf, R. Buhrman, and D. Ralph, Nature (London) 425, 380 (2003).

${ }^{7}$ S. Kaka, M. Pufall, W. Rippard, T. Silva, S. Russek, and J. Katine, Nature (London) 437, 389 (2005).

${ }^{8}$ F. Mancoff, N. Rizzo, B. N. Engel, and S. Tehrani, Nature (London) 437, 393 (2005).

${ }^{9}$ A. N. Slavin and V. S. Tiberkevich, Phys. Rev. B 74, 104401 (2006).

${ }^{10}$ M. R. Pufall, W. H. Rippard, S. E. Russek, S. Kaka, and J. A. Katine, Phys. Rev. Lett. 97, 087206 (2006).

${ }^{11}$ A. N. Slavin and V. S. Tiberkevich, Phys. Rev. B 72, 092407 (2005).

${ }^{12}$ J. Grollier, V. Cros, and A. Fert, Phys. Rev. B 73, 060409(R) (2006).

${ }^{13}$ I. Krivorotov, N. Emley, J. Sankey, S. Kiselev, D. Ralph, and R. Buhrman, Science 307, 228 (2005).

${ }^{14}$ A. N. Slavin and V. S. Tiberkevich, Phys. Rev. B 72, 094428 (2005).

${ }^{15}$ Z. Li, Y. C. Li, and S. Zhang, Phys. Rev. B 74, 054417 (2006).

${ }^{16}$ J. Tejada, E. Chudnovsky, J. Hernandez, and R. Amigo, Appl. Phys. Lett. 84, 2373 (2006).

${ }^{17}$ A. Hernandez-Miguel, M. Jordi, R. Amigo, A. Garcia-Santiago, and J. Hernandez, Europhys. Lett. 69, 270 (2005).

${ }^{18}$ E. M. Chudnovsky and D. A. Garanin, Phys. Rev. Lett. 89, 157201 (2002).

${ }^{19}$ P. D. Ye, D. Weiss, R. R. Gerhardts, M. Seeger, K. von Klitzing, K. Eberl, and H. Nickel, Phys. Rev. Lett. 74, 3013 (1995).

${ }^{20}$ R. Dicke, Phys. Rev. 93, 99 (1954).

${ }^{21}$ M. Gross and S. Haroche, Phys. Rep. 93, 301 (1982).

${ }^{22}$ A. Gover, Phys. Rev. Lett. 96, 124801 (2006).
${ }^{23}$ C. L. Joseph, C. Calero, and E. M. Chudnovsky, Phys. Rev. B 70, 174416 (2004).

${ }^{24}$ M. G. Benedict, P. Foldi, and F. M. Peeters, Phys. Rev. B 72, 214430 (2005).

${ }^{25}$ B. Draine and A. Lazarian, Astrophys. J. 494, L19 (1998).

${ }^{26}$ B. Draine and A. Lazarian, Astrophys. J. 494, 740 (1999).

${ }^{27}$ A. Nogaret, Phys. Rev. Lett. 94, 147207 (2005).

${ }^{28}$ A. Nogaret, S. J. Bending, and M. Henini, Phys. Rev. Lett. 84, 2231 (2000).

${ }^{29}$ D. N. Lawton, A. Nogaret, S. J. Bending, D. K. Maude, J. C. Portal, and M. Henini, Phys. Rev. B 64, 033312 (2001).

${ }^{30}$ A. Nogaret, D. N. Lawton, D. K. Maude, J. C. Portal, and M. Henini, Phys. Rev. B 67, 165317 (2003).

${ }^{31}$ M. Hara, A. Endo, S. Katsumoto, and Y. Iye, Phys. Rev. B 69, 153304 (2004)

${ }^{32}$ V. Kubrak, F. Rahman, B. Gallagher, P. Main, M. Henini, C. Marrows, and M. Howson, Appl. Phys. Lett. 74, 2507 (1999).

${ }^{33}$ A. Matulis, F. Peeters, and P. Vasilopoulos, Phys. Rev. Lett. 72, 1518 (1994).

${ }^{34}$ J. Reijniers and F. M. Peeters, Phys. Rev. B 63, 165317 (2001).

${ }^{35}$ D. K. K. Lee, J. T. Chalker, and D. Y. K. Ko, Phys. Rev. B 50, 5272 (1994).

${ }^{36}$ F. Evers, A. D. Mirlin, D. G. Polyakov, and P. Wölfle, Phys. Rev. B 60, 8951 (1999).

${ }^{37}$ A. Nogaret and F. M. Peeters, preceding paper, Phys. Rev. B 76, 075311 (2007).

${ }^{38}$ C. Cohen-Tannoudji, J. Dupont-Roc, and G. Grynberg, Atom Photon Interactions, Basic Processes and Applications (WileyVCH, Weinheim, 2004).

${ }^{39}$ N. Bloembergen and R. Pound, Phys. Rev. 95, 8 (1954).

${ }^{40}$ A. Tackeuchi, O. Wada, and Y. Nishikawa, Appl. Phys. Lett. 70, 1131 (1997).

${ }^{41}$ A. Tackeuchi, S. Muto, T. Inata, and F. Fujii, Appl. Phys. Lett. 56, 2213 (1990).

${ }^{42}$ J. Kikkawa, I. Smorchkova, N. Samarth, and D. Awschalom, Science 277, 1284 (1997).

${ }^{43}$ K. Halbach, Nucl. Instrum. Methods Phys. Res. 187, 109 (1981).

${ }^{44}$ J. Jackson, Classical Electrodynamics, 3rd ed. (Wiley, New York, 1998).

${ }^{45}$ P. Duke, Synchrotron Radiation (Oxford University Press, Oxford, 2000). 